\title{
Representaciones sociales acerca del proceso de Escritura Académica: el caso de la tesis en una Licenciatura en Historia*
}

\author{
Social representations regarding the academic writing process: \\ the case of the thesis for the bachelor's degree in history \\ Representações sociais sobre o processo de escritura acadêmica: \\ o caso da tese numa licenciatura em história
}

\author{
Juana Marinkovich, Juan Salazar
}

Pontificia Universidad Católica de Valparaíso, Chile

Teléfono: (56-32) 2273439. E-mail: jmarinko@ucv.cl

\begin{abstract}
RESUMEN
El estudio acerca del proceso de escritura, sobre todo en el ámbito académico, se ha transformado, en las últimas décadas, en un objeto de investigación recurrente. En este sentido, interesa centrarse en las concepciones que tienen los profesores respecto del proceso de escritura de una tesis de Licenciatura. El objetivo que guía este estudio es dar cuenta de las representaciones sociales que manifiesta un grupo de académicos de un programa de Licenciatura en Historia de una Universidad del Consejo de Rectores de Chile. Para cumplir con este propósito, se utilizó la metodología de la Teoría Empíricamente Fundada (TEF) que permite, a partir de un muestreo teórico, profundizar en los datos recabados a través de las entrevistas realizadas a los sujetos en cuestión. Los datos una vez sometidos a todas las instancias de codificación se sintetizan en una teoría emergente cuyos ejes son, por una parte, la dialogicidad entre los actores del proceso de escritura de la tesis (profesor guía/ alumno tesista); y, por otra, la recursividad en la gestación de dicho género a lo largo de los diferentes estadios de desarrollo del mismo, reflejada en la revisión y en el monitoreo del proceso. Por último, el género tesis en este contexto se ubica al término de la formación inicial y constituye parte del itinerario que conduce al estudiante a los estudios de postgrado, en donde se espera que se inserte con mayor propiedad en la comunidad discursiva especializada.
\end{abstract}

Palabras clave: representaciones sociales, alfabetización académica, escritura académica, género tesis.

\begin{abstract}
The study of the writing process, especially in the academic field, has become a recurring subject of research in recent decades. In this sense, our interest focuses on the conceptions that professors have about the process of writing a bachelor's thesis. The aim of this research is to account for the social representations manifested by a group of professors from an undergraduate History program at a Traditional Chilean University. To fulfill this purpose, we used the methodology of the Grounded Theory (GT). This theory allows deepening into the data collected through interviews, starting from a theoretical sampling. The data were submitted to all levels of coding, and were synthesized in an emerging theory whose axis are, first, the dialogue between the actors of the process of writing a thesis (academic adviser / dissertator student) and, secondly, the resourcefulness in the gestation of this genre throughout the various stages of development of the same, as reflected in the review and monitoring of the process. Finally, the thesis genre in this context is allocated at the end of the initial academic training. It is a part of the itinerary that leads the student to postgraduate studies, where he or she is expected to fit in with greater property into the specialized discourse community.
\end{abstract}

Key words: social representations, academic literacy, academic writing, thesis as genre.

* $\quad$ Proyecto FONDECYT 1090151: "La escritura en las disciplinas en la Universidad: El caso de la Licenciatura en Ciencias y Humanidades". 


\title{
RESUMO
}

O estudo do processo de escrita, especialmente na academia, se transformou nas últimas décadas, num tema recorrente da pesquisa. Neste sentido, o interesse centrou-se nas concepções que os professores têm sobre o processo de escrever uma tese de licenciatura. O objetivo principal deste estudo é dar conta das representações sociais manifestadas por um grupo de acadêmicos de um programa de Licenciatura em História de uma Universidade do Conselho de Reitores do Chile. Para cumprir este propósito, foi utilizada a metodologia da teoria empiricamente fundamentada (TEF), que permite, a partir de uma amostragem teórica, aprofundar os dados coletados através de entrevistas com os sujeitos em questão. Os dados, uma vez submetidos a todos os níveis de codificação, são sintetizados numa teoria emergente, cujos eixos são, em primeiro lugar, o diálogo entre os atores do processo de escritura da tese (professores orientadores / aluno tesista) e, por outro, a recursividade na gestação deste gênero em todas as fases do seu desenvolvimento, que se traduzem na revisão e no acompanhamento do processo. Finalmente, neste contexto, o gênero tese está localizado no final da formação inicial e faz parte do itinerário que leva o estudante aos estudos de pós-graduação, esperando-se que seja inserido com maior propriedade na comunidade de discurso especializado.

Palavras-chave: representações sociais, alfabetização acadêmica, escrita acadêmica, teoria de gênero.

\section{INTRODUCCION}

Numerosas investigaciones han intentado dar cuenta de los problemas que surgen cuando los estudiantes deben adquirir una nueva alfabetización, denominada alfabetización académica, aquella que es propia y particular de cada ámbito disciplinario y de la que deberán apropiarse en su formación universitaria (Treet 1999; Narvaja de Arnoux 2006; Carlino 2002; Di Stefano 2003, entre otros). Al respecto, Carlino (2005: 13) define la alfabetización académica como:

\begin{abstract}
"las nociones y estrategias necesarias para participar en la cultura discursiva de las disciplinas así como en actividades de producción y análisis de textos requeridos para aprender en la universidad (...); apunta a las prácticas de lenguaje y pensamiento propias del ámbito académico superior".
\end{abstract}

Un aspecto importante a considerar en esta materia es que el grado de desarrollo de las capacidades escriturales determina, en gran medida, el acceso y la permanencia en la enseñanza superior, toda vez que es en este espacio o en esta cultura que el discurso escrito se ha convertido en una herramienta de poder, de inserción y de reconocimiento (Barthes 2003). A lo que Petrucci (2002: 27) agrega: "la escritura [...] instaura, donde quiera que aparezca, una relación tajante y fuerte de desigualdad entre aquel que escribe y aquel que no; entre aquel que lee y aquel que no, entre el que lo hace bien y mucho y el que lo hace mal y poco; y esta desigualdad sigue y revela a la vez los límites de la distribución social de la riqueza, de la diferencia de sexos, edades, geografías y culturas".

Sin embargo, no basta un diagnóstico de esta realidad ni tampoco la aplicación de estrategias de intervención didáctica. Se hace necesario, entonces, entre otras interrogantes, conocer en profundidad qué concepciones o qué representaciones sociales evidencian los docentes y los alumnos acerca de este proceso de alfabetización académica, cómo conciben el proceso de escritura académica o qué géneros discursivos deben escribir.

Algunas investigaciones en lengua española han aportado en este sentido, como es el caso, entre otros, de Castelló (2000) en España, quien se centra en las concepciones de los estudiantes de nivel secundario sobre el proceso de escritura; en Argentina, Giménez, Ortiz y Sánchez (2000) realizan un acercamiento a las representaciones de los 
adolescentes respecto de la escritura y Pereira y Di Stefano (2007) abordan las representaciones sociales e interacción entre pares en un taller de escritura en postgrado; en Chile, también encontramos trabajos señeros; por una parte, la investigación de Meneses (2008) en cuanto a las representaciones discursivas sobre leer y escribir de los diferentes agentes educativos en las áreas prioritarias del currículum de quinto año de enseñanza básica (alumnos entre 10 y 11 años); y por otra, la tesis doctoral de Rubio (2008) acerca de la evolución de las concepciones sobre el proceso de producción escrita de estudiantes durante su formación como maestros.

Plantearnos, entonces, desde la perspectiva de las representaciones sociales de los diferentes agentes educativos en la educación superior sobre la escritura académica, no solo permitirá ofrecer programas de enseñanza acordes con los intereses y necesidades de nuestros estudiantes, sino también responder a una nueva visión de la escritura en la educación superior que, como declara Carlino (2003), sugiere que los profesores universitarios conciban la relación de la enseñanza de las prácticas letradas con la construcción de saberes específicos (principalmente, contenidos o conceptos).

En este contexto, el estudio se orienta a responder a la pregunta guía: ¿cómo se representan socialmente los académicos de una universidad chilena el proceso de escribir una tesis de Licenciatura en Historia? Para ello se emplean herramientas analíticas que nos proporciona la metodología de Teoría Empíricamente Fundada (TEF), las que permiten, en forma progresiva y cada vez más profunda, aplicar las diversas codificaciones de los datos hasta conformar una teoría emergente del fenómeno en cuestión. De este modo, los resultados obtenidos muestran el gran potencial investigativo de esta metodología al promover la reflexión y la continua mirada de los datos hasta conseguir su saturación.

Finalmente, en los apartados siguientes se despliega el marco de referencia, constituido por las representaciones sociales y la escritura, la escritura académica y la tesis como género discursivo. A continuación se describen el marco metodológico y los resultados obtenidos luego de la aplicación de las distintas codificaciones para, posteriormente, cerrar con las reflexiones finales.

\section{MARCO DE REFERENCIA}

\subsection{REPRESENTACIONES SOCIALES (RS)}

Para Vasilachis (1997: 301), las RS corresponden a "construcciones simbólicas individuales y/o colectivas a las que los sujetos apelan o que los sujetos crean para interpretar el mundo, para reflexionar sobre su propia situación y la de los demás y para determinar el alcance y la posibilidad de su acción histórica".

Las RS se construyen, entonces, a partir de las experiencias humanas, la comunicación interpersonal, los modos de pensamiento, las tradiciones y la formación. Es decir, se trata de "un conocimiento socialmente elaborado y compartido" (Jodelet 1984: 473).

Por su parte, Farr (1984) señala que las RS, desde una perspectiva esquemática, aparecen cuando los individuos debaten temas de interés mutuo o cuando existe el eco de los acontecimientos seleccionados como significativos o interesantes para quienes tienen el control de los medios de comunicación o de la estabilidad social (educadores, políticos, empresarios, etc.). Agrega, además, que las representaciones sociales tienen 
una doble función. Por un lado, hacer que lo extraño resulte familiar; y, por otro, que lo invisible sea perceptible, ya que lo insólito o lo desconocido son amenazantes cuando no se tiene una categoría para clasificarlos.

Las RS manifestarían los modos en que los sujetos, formando parte de un grupo social, construyen explicaciones de lo real, fragmentando la realidad o reteniendo, mediante el lenguaje u otra manifestación simbólica, aquello que es necesario para la creación de sentido (Meneses, 2008).

Siguiendo a Castorina (2003), lo que permite calificar las representaciones como 'sociales' es, precisamente, el hecho de que se elaboran durante los intercambios e interacciones comunicativas.

Es importante señalar que las representaciones sociales siempre hacen referencia a un objeto, no existen en abstracto sino que constituyen la manera en que los individuos interpretan, piensan, conciben y explican un fenómeno, un concepto o una práctica (Bourdieu, 1997).

Las RS se convierten, en nuestro caso, en expresiones de lo que los docentes de la Licenciatura en Historia han formulado acerca de la escritura académica y del proceso que esta conlleva cuando los alumnos se ven enfrentados a escribir la tesis de grado. Estamos conscientes de que durante las entrevistas sostenidas con estos docentes surgen variables de orden experiencial, valórico, emocional y motivacional, que, en definitiva, constituyen las representaciones sociales del fenómeno en estudio.

\subsection{ESCRITURA ACADEMICA}

La escritura en el ámbito académico constituye el medio privilegiado que utilizan las comunidades disciplinares para la producción y comunicación del conocimiento especializado. Esta aseveración supone, en cierta manera, tomar una postura, por un lado, epistémica del proceso de escritura, tal como la visualizan Bereiter y Scardamalia (1987) en los modelos explicativos de "decir el conocimiento" y el de "transformar el conocimiento", constituyéndose este último en el desideratum de un escritor experto que es capaz de establecer objetivos y problematizar la tarea de escritura en una dialéctica constante entre el espacio discursivo y el espacio temático, sometidos ambos a un control consciente. Esto redundará, en definitiva, en un producto escrito maduro.

Por otro lado, si consideramos que cada comunidad genera y transmite el conocimiento de acuerdo con ciertas prácticas o convenciones discursivas o como lo declara Bajtín (1982: 248): "cada esfera del uso lingüístico elabora sus tipos relativamente estables de textos, a los que denominamos géneros discursivos", debemos coincidir, entonces, con Hyland (2003: 21) que señala:

"El género se refiere a las diversas formas abstractas y sociales de usar el lenguaje. (Sus características) depende(n) del contexto social en que se ha creado y en que se usa, y (...) podemos describir estas características para agrupar los textos parecidos entre sí y para determinar las elecciones y las restricciones que debe afrontar el autor al escribirlos. De este modo, se concibe el lenguaje como algo incrustado en la realidad social y como algo constitutivo de la misma. Es a través del uso recurrente de formas convencionalizadas del lenguaje como los individuos desarrollan sus relaciones, se establecen comunidades y se realizan las cosas" (Hyland 2003: 21). 
Esta noción de género nos remite, según Hyland (2002), a dos vertientes dentro de los enfoques de escritura que él denomina centrados en el lector. La primera entiende la escritura como interacción social, en que el escritor se dirige a un posible lector, teniendo en cuenta sus intereses y necesidades. La segunda se centra en la escritura como construcción social, es decir, interesa el escritor como miembro de una comunidad y las elecciones retóricas que debe realizar como tal. A nuestro juicio, ya Linda Flower en sus trabajos de 1989 y 1994 postulaba una teoría interactiva de la producción escrita que integrara la cognición y el contexto desde una perspectiva sociocognitiva; aún más, concebía al escritor en acción en contextos determinados, negociando significados con un potencial lector.

\subsection{LA TESIS COMO GENERO DISCURSIVO}

De los tipos de géneros discursivos que circulan al alero de las instituciones de educación superior, tales como apuntes, pruebas, exámenes, informes de investigación, tesinas, nuestro foco está puesto en las tesis que se escriben al término del grado de la Licenciatura en Historia en el contexto de una universidad chilena, perteneciente al Consejo de Rectores.

La tesis como género discursivo académico ha sido poco abordado con respecto al nivel de formación inicial, no así en postgrado. Incluso, se le ha dado mayor énfasis a estudios respecto de otros textos académicos o los que dan a conocer las investigaciones de los especialistas, v.g., Artículos de Investigación. Prueba de ello es la amplia bibliografía que existe en el ámbito (Swales 1981, 1990, 2004; Bathia 2002; Kanoksilapatham 2003, 2005; Venegas 2006; entre muchos otros).

Las tesis han sido, más bien, estudiadas desde la practicidad. Los manuales de escritura de tesis son abundantes (Calderón y Bono 2003) y una simple búsqueda en la web arroja más de 170.000 resultados. Uno de estos sitios la define como un informe que concierne a un problema o conjunto de problemas en un área definida de la ciencia y explica lo que se sabe de él previamente, lo que se haría para resolverlo, lo que sus resultados significan, y dónde o cómo se pueden proponer proyecciones, más allá del campo delimitado por el trabajo.

Asimismo, Camps (2007: 11) expresa que "el destinatario de una tesis es la comunidad científica con diversas tradiciones según los campos del saber” y que en la elaboración de la misma "el autor debe aprender a encontrar su voz sin dejar de mostrar la multiplicidad de voces que se han ocupado con anterioridad del tema". La misma autora señala que escribir una tesis de grado significa construir y transformar el conocimiento.

Ahora bien, solo recientemente se ha instalado una preocupación por los géneros académicos $\mathrm{y}$, en particular, por el género tesis, el que ha sido presentado por Parodi (2008: 66) en tanto:

"Género discursivo que tiene como macropropósito persuadir acerca de un planteamiento teórico o ideológico. Idealmente, circula en el ámbito científico y la relación entre los participantes es entre escritor experto y lector experto".

Aunque es una definición breve y general, resulta un primer apresto a la problemática de este género, que ya ha sido investigada a nivel de postgrado. Al respecto, Pereira y Di 
Stefano (2007) realizan un análisis de las percepciones de los estudiantes de postgrado respecto de la escritura de su proyecto de tesis y Hermosilla y Villalobos (2006) narran el proceso de formación de un investigador, el cual pareciera iniciarse con la escritura de la tesis de grado.

Por último, Paltridge y Starfield (2007) han determinado ocho características de una tesis de grado, las que se centran en el estudio del contexto, la identificación de los lectores potenciales y el seguimiento de los patrones retórico-lingüísticos de la comunidad en la que se escribe y publica la tesis.

\section{Figura 1}

Características de una tesis de grado.

(Basado en Paltridge y Starfield, 2007)

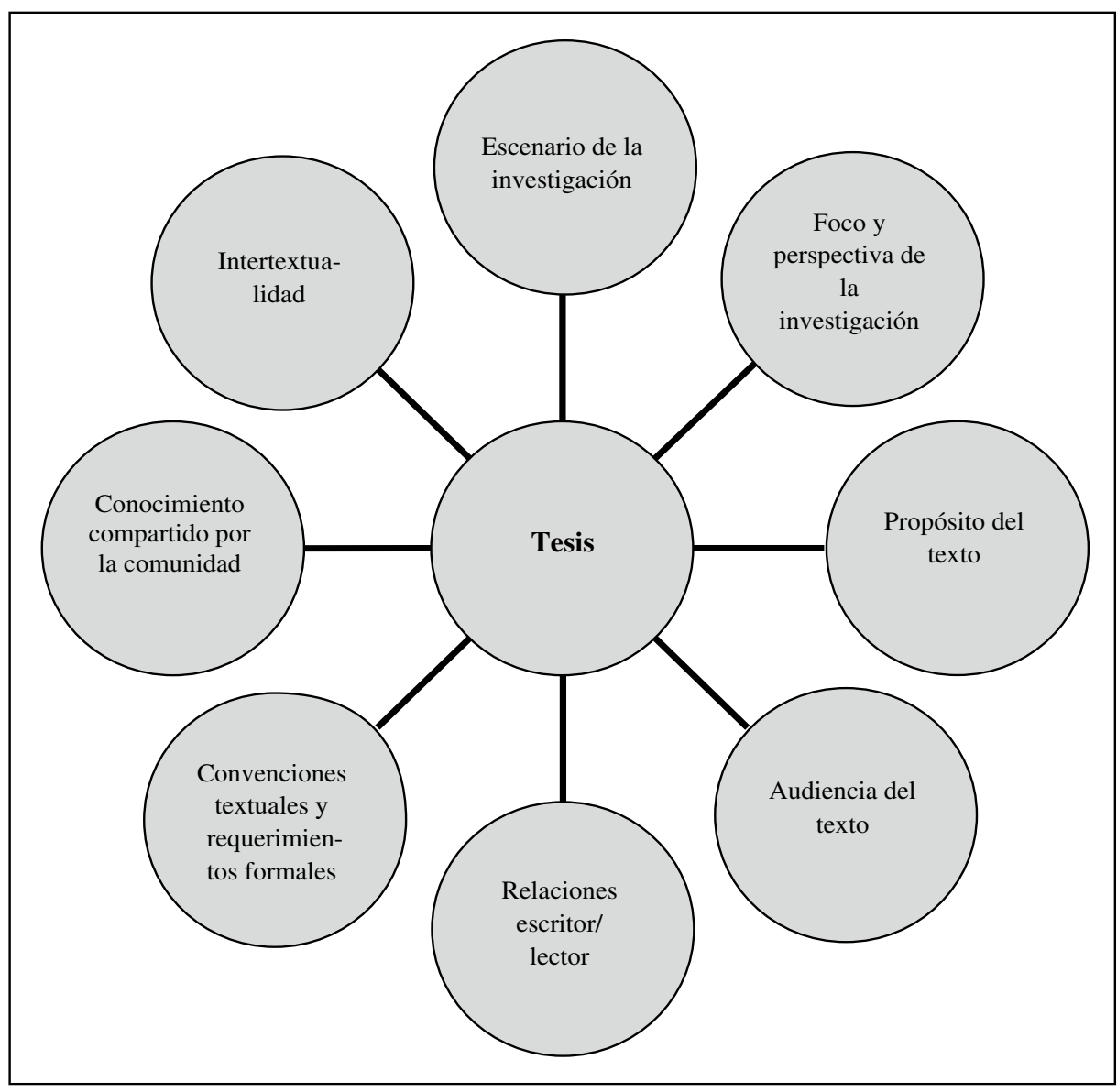


La tesis se convierte, entonces, en la justificación de conocimientos sobre un tema específico, dentro de la gama de áreas, temas y materias que integran el currículo de un programa de formación. Asimismo, permite, a través de una investigación formal, realizar una aportación, recopilación y/o experimentación de un conocimiento, tema o disciplina específica, dentro de una carrera profesional.

\section{MARCO METODOLOGICO}

\subsection{TIPO DE INVESTIGACION}

La investigación que hemos emprendido es de naturaleza cualitativa, exploratoria $\mathrm{y}$, fundamentalmente, inductiva. El marco en el que esta se inscribe es el paradigma interpretativo, cuyo foco son los significados que las personas atribuyen a la realidad en la que están insertas (Krause, 1995), lo que coincide con los estudios acerca de las representaciones sociales, aspecto que nos ocupa en este artículo.

Ahora bien, adherir a este paradigma significa, desde el punto de vista epistemológico, poner el acento en la subjetividad y, desde el punto de vista metodológico, considerar al investigador como un sujeto que puede acceder con propiedad a la realidad investigada, utilizando para ello técnicas que le permitan interactuar con los participantes en la investigación.

Asimismo, en la búsqueda de una metodología de análisis cualitativo, se ha optado por la TEF, en la que las hipótesis y teorías emergen desde los datos y en los que se pueden descubrir aquellos aspectos que son relevantes para el estudio de los procesos sociales (Strauss y Corbin, 1997; Suddaby, 2006).

La aportación más relevante de la TEF hace referencia a su poder explicativo y a la emergencia de significados a partir de los datos recogidos que, en conjunto, permitirían, en nuestro caso, ofrecer una explicación de las representaciones sociales que los profesores manifiestan acerca del proceso de escritura de una tesis en el ámbito de la Licenciatura en Historia.

Esta opción no sólo se circunscribe al presente estudio, sino que también constituye la base metodológica del proyecto de investigación mayor en el cual se inserta este trabajo.

\subsection{PREGUNTAS DE INVESTIGACION}

\subsubsection{Pregunta inicial}

¿Cómo se representan socialmente el proceso de escritura de una tesis de Licenciatura en Historia los académicos que guían dichas tesis?

\subsubsection{Preguntas directrices}

¿Qué rol le asignan estos académicos a la escritura de este género?

¿Qué características son propias de una tesis de grado en esta especialidad?

¿Qué fases se contemplan en el desarrollo de una tesis de grado en la especialidad? 


\subsection{TIPO DE MUESTREO}

El tipo de muestreo, si somos congruentes con la TEF, se denomina muestreo teórico, mediante el cual se selecciona la muestra en etapas sucesivas: se eligen los primeros sujetos, documentos o situaciones y se analizan los datos obtenidos, desarrollando conceptos, categorías e hipótesis y, a partir de estos, se generan criterios que permitirán elegir los sujetos u otras fuentes de información que se integrarán posteriormente. En nuestro caso, damos cuenta de los datos recabados con los primeros sujetos, profesores pertenecientes a la comunidad disciplinar de la Licenciatura en Historia de una institución de Educación Superior en Chile, que teóricamente corresponden a la muestra total, es decir, aquellos profesores que guían tesis de grado, sin distinción de años de servicio, ni de las temáticas abordadas en dichas tesis. La decisión de tomar los datos hasta ahora obtenidos obedece a que, a nuestro juicio, podemos ya avanzar ciertas hipótesis y conformar un esbozo de una teoría emergente, que permita caracterizar el proceso de escribir una tesis de grado según los profesores, actores sociales que, junto a los alumnos, constituyen la comunidad disciplinar en cuestión.

\subsection{TECNICA DE RECOLECCION DE DATOS}

Una vez realizada la entrada al campo a través de los contactos con directivos, académicos y personal administrativo y reglamentaciones propias de la Unidad Académica, se procedió a agendar las entrevistas semiestructuradas en profundidad que se aplicaron en forma individual a los docentes que habían guiado tesis de grado en Licenciatura en Historia durante los años 2007 y 2008. Se tuvo especial cuidado para que las entrevistas fueran lo más distendidas posibles y se asemejaran a una actividad habitual para los profesores. Asimismo, la entrevista a cada profesor se realizó en el contexto académico en el cual está inserto, especialmente en su oficina de trabajo. La duración de la entrevista fue en promedio de 60 minutos y mientras esta se llevaba a cabo se tomaron las notas de campo respectivas.

\subsection{METODOLOGIA DE ANALISIS}

Para resolver la pregunta inicial y las preguntas directrices, las entrevistas, previamente transcritas, y complementadas por las notas de campo, se analizaron, tal como ya se expresó, según la metodología propuesta por la TEF.

La TEF utiliza una serie de procedimientos que, por medio de la interpretación, permite generar una teoría explicativa de un fenómeno en estudio. En este sentido, los conceptos y las relaciones entre los datos son producidos y examinados mediante el método comparativo constante y los criterios del muestreo teórico hasta la saturación conceptual de las categorías encontradas (Strauss y Corbin, 1990; Glaser, 1992).

Ahora bien, para proceder al análisis, se inicia el proceso de codificación que se realiza en tres fases: abierta, axial y selectiva.

En la codificación abierta, se fragmentaron los datos, relevando conceptos y sus propiedades e integrándolos en un conjunto de categorías. A medida que surgen nuevas categorías, estas se ajustan a las categorías ya existentes hasta saturar los datos. Estas categorías comienzan a mostrar teóricamente fenómenos, procesos, modelos, causas, etc., 
que en la codificación axial encontrarán su delimitación en términos de un código como variable central, que se traduce en una categoría nuclear.

En la codificación axial se rearticulan los datos que fueron fragmentados y clasificados intuitivamente en la codificación abierta, relacionando las categorías y subcategorías en función de una categoría eje. De este modo, se intenta responder a lo que está sucediendo (fenómeno), a las condiciones (causales, intervinientes y contextuales) y a las acciones/ interacciones (situaciones, rutinas, intenciones, etc.). Asimismo, todos los componentes de la codificación axial se agrupan y se relacionan jerárquicamente o gradualmente en forma lineal o recursiva en torno al eje central (Spiggle, 1994).

Finalmente, la codificación selectiva, entendida como "el proceso de integrar y refinar la teoría" (Strauss y Corbin, 2002: 157), favorece, por una parte, la integración de las categorías principales para formar un esquema teórico (teoría que se aplica a todos los casos) alrededor de una categoría central (interpretación principal sobre el tema de investigación), y, por otra, la lógica interna de la teoría, la densidad de las categorías y la reducción de las mismas, como también su validación.

\section{ANALISIS E INTERPRETACION DE LOS DATOS}

En este apartado se presenta el análisis e interpretación de los resultados de este estudio. En primer lugar, se muestra la codificación axial de dos fenómenos tratados, antecedida de las interrogantes que surgieron al categorizar los conceptos en la codificación abierta. Finalmente, en un intento de codificación selectiva, se esquematizan conjuntamente las categorías con tal de dar respuesta al fenómeno del proceso de escritura de una tesis de Licenciatura en Historia.

\subsection{HACIA UNA CATEGORIA NUCLEAR Y SUS RELACIONES}

Tomando las fases de la metodología de análisis anteriormente descrita, los códigos obtenidos del examen y fragmentación realizados en la codificación abierta se fueron agrupando conceptualmente en nuevos códigos que comienzan a dar forma a la teoría que explica lo que sucede con los datos. Esto permite extraer las percepciones y representaciones sociales y reiterativas del discurso de los profesores que guían tesis en la Licenciatura en Historia.

Enseguida, como parte de la codificación axial, los códigos se han organizado jerárquicamente, lo que ha permitido el surgimiento de ciertas interrogantes fundamentales, que se desprenden tanto de la pregunta inicial como de las preguntas directrices del estudio:

1. ¿Qué es una tesis de Licenciatura en Historia?

2. ¿Qué sucede con el proceso de escritura de una tesis de Licenciatura en Historia?

- ¿Quién monitorea?

- ¿Cómo se accede a los temas de escritura de la tesis?

- ¿Qué procesos se siguen? 
De este modo, el fenómeno de la escritura de una tesis supondría, en primer lugar, la caracterización de este género y, en segundo lugar, el establecimiento del proceso de escritura de la tesis y el rol de los actores de dicho proceso.

\subsubsection{Rasgos fundamentales del género tesis de Licenciatura en Historia}

En la figura 2 se muestran los resultados de la codificación axial para el primer fenómeno, es decir 'Características del género tesis en la Licenciatura en Historia'.

\section{Figura 2}

Codificación axial del fenómeno 'Características del género tesis en la Licenciatura en Historia'

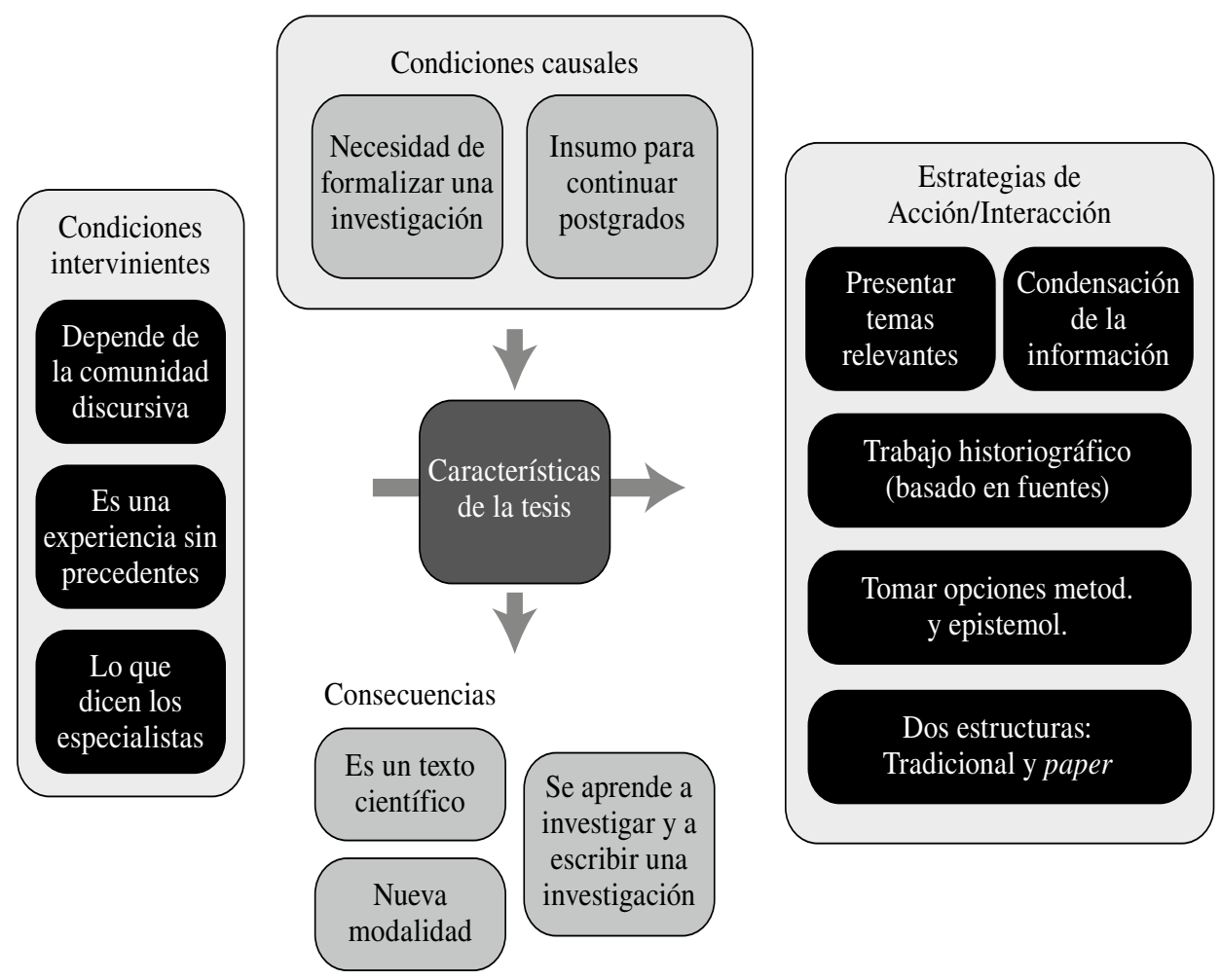

En cuanto a la caracterización de la tesis de grado, los profesores señalan que se escribe una tesis como una manera de formalizar una investigación científica: "Si pensamos en la estructura curricular de la Licenciatura en Historia, el trabajo más importante de formalización de una investigación es la tesis”. Esta sistematización permitiría continuar estudios de postgrado, "porque si ese estudiante [...] se supone que está estudiando licenciatura, por lo tanto, su camino es la investigación que tendría que ser investigar 
y publicar. Continuar, además, sus estudios de postgrado”. A su vez, este género no se concibe como independiente del contexto, sino que, por el contrario, depende de la comunidad discursiva en la que se produce y se convierte en un deber para el escritor: "Formalizar esa investigación en los términos que la comunidad científica dice que debe formalizarse un resultado de investigación".

Todos los académicos registran en sus intervenciones que este género no tiene precedentes en la formación curricular de los estudiantes y, por lo mismo, obedece a criterios establecidos por los profesores guía en cuanto a su estructura y a las temáticas que se aborden. Tal como ellos mismos afirman: "Comúnmente el procedimiento de elección de las temáticas de los trabajos depende muchos de sus propias experiencias e inquietudes [de los estudiantes], y ellos asumen a los profesores que son especialistas. Siempre hay un especialista para ese tema". Respecto de la estructura, también se señala: "yo les entrego una estructura que, en el fondo, es la estructura tradicional, pero le hago hincapié a cómo tiene que ir hasta las normas de citas a pie de página, etc.”.

Para llevar a cabo la producción escrita de este género, los informantes se representan una variedad de estrategias de acción/interacción, bajo un determinado conjunto de condiciones contextuales (causales e intervinientes), sin las cuales no hubiera sido posible concebir estos códigos. Así, este género presentaría temas relevantes para el quehacer de la comunidad científica de Historia y adoptaría el método historiográfico, un método comparativo basado en fuentes primarias o secundarias, directas o indirectas. Mientras unos señalan, por ejemplo, "yo creo que un historiador se forma leyendo archivos, no se forma leyendo libros, se forma con las fuentes, con los datos", otros, en cambio, adhieren al acceso a nuevas fuentes ("esto es un paper, y el foco está puesto en un tema actual y, por lo tanto, un tema actual que tiene cifras, números, datos cuantitativos, cómo se trabaja en datos cuantitativos, la consistencia metodológica"). Cualquiera sea la opción de estos métodos, este no estará reñido con el método científico, solo diferirá del de las ciencias exactas o experimentales en que las tácticas para la resolución de sus problemas son específicas a su campo de investigación.

Esto se corrobora en el hecho de que en la presentación escrita de la tesis se exige precisión y condensación, rasgo característico de la conformación de un texto científico (Albentosa y Moya, 2000). A su vez, los mismos académicos han manifestado la existencia de dos tipos de formato para una tesis en este ámbito disciplinar: uno de tipo tradicional o lo que siempre se ha exigido como resultado de un proceso de formación inicial en el ámbito académico y otro en formato paper, cercano al artículo científico. Realizar esta elección, es decir, escoger el formato de presentación de la tesis no es tarea del estudiante, sino que, coherente con las condiciones intervinientes, depende de los criterios del profesor guía. Además, esta evidencia supone una toma de posición epistemológica y metodológica. Uno de los profesores, a modo de ejemplo, señala que en la escritura de la tesis es "donde los alumnos, finalmente, aprenden a investigar y aprender a escribir un texto de investigación" y, por ello, "en un trabajo de ese tipo no es solamente ver el texto, sino que el texto implica una opción metodológica, una opción epistemológica", esto es, una opción científica, lo que da cuenta de la complejidad de las redes de construcción de conocimientos y significados al interior de esta comunidad disciplinar (Flores, 2008).

Como síntesis de lo descrito, se puede señalar que la tesis se conforma como un texto científico que, en el escenario de esta comunidad discursiva, ha ido generando 
nuevas estructuras (tradicionales y tipo paper). Al escribir una tesis de Licenciatura en Historia habría consenso en que se aprende a investigar en la disciplina, como también a escribir lo que se investiga, tomando en consideración todas las condiciones y estrategias previamente señaladas.

\subsubsection{Fases del proceso de escritura de una tesis de Licenciatura en Historia}

Ahora bien, para el desarrollo del proceso de escritura de la tesis en la Licenciatura en Historia se presentan los componentes del fenómeno gráficamente separados, dadas la extensión de las explicaciones y de las categorías que en ellos se insertan.

Entre las condiciones causales del proceso de escritura de la tesis se encuentra la intención de la comunidad por insertar rápidamente al estudiante en el ámbito académico y profesional. Asimismo, en cuanto a la sistematización de la investigación historiográfica, valen las mismas categorías formuladas para el primer fenómeno, ya que lo que está en juego es el proceso de escritura. Además, como parte de la instauración de una cultura universitaria, la tesis se ha convertido en el documento oficial que les permite a los tesistas optar a su respectivo grado académico, haciendo de la producción de este género una exigencia institucional (ver figura 3).

\section{Figura 3}

\section{Condiciones causales de la codificación axial del fenómeno \\ 'Proceso de escritura de la tesis'}

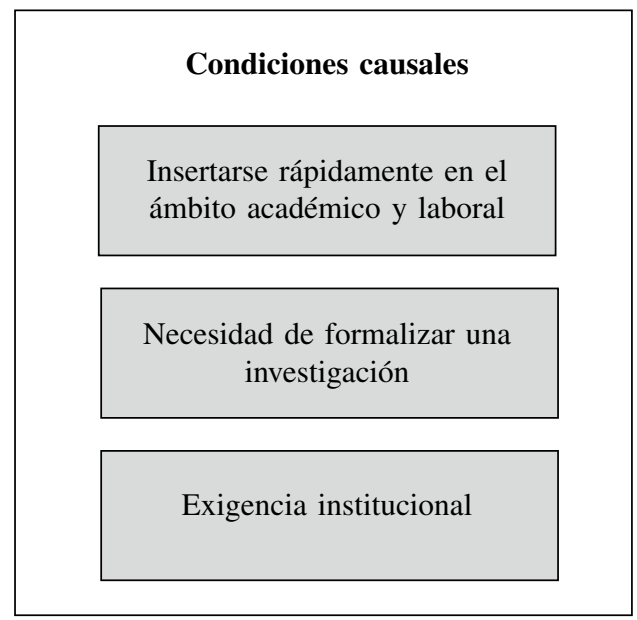

Respecto de las condiciones que intervienen en la realización del proceso de escritura de la tesis, de acuerdo a la figura 4, las pautas que el profesor entrega resultan de gran aporte y son fundamentales para que el tesista determine las acciones que emprenderá en la producción de su investigación. 
Figura 4

Condiciones intervinientes de la codificación axial del fenómeno 'Proceso de escritura de la tesis'

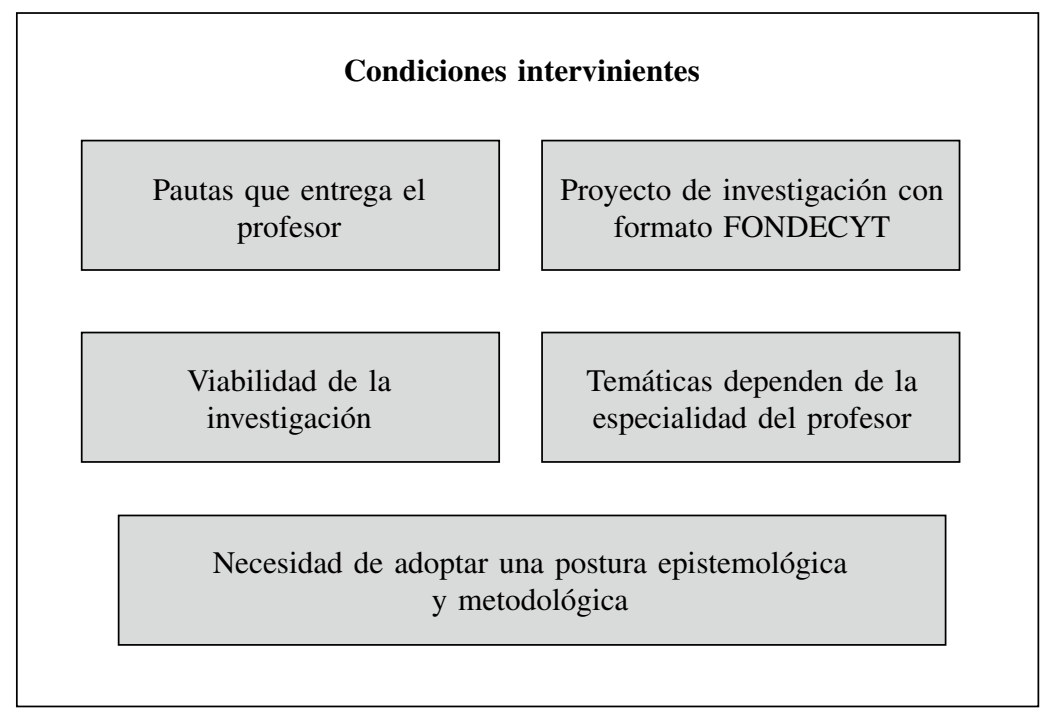

De este modo, el profesor evalúa la viabilidad de la investigación siguiendo, entre otros, los parámetros de los proyectos de investigación del Fondo Nacional de Desarrollo de la Ciencia y la Tecnología (FONDECYT): "Hoy día un estudiante, en el grupo nuestro, que quiere hacer una investigación, tiene que formular la investigación en las pautas de un proyecto FONDECYT".

Se afirma que la comunidad de especialistas, en general, es determinante para el proceso de escritura de la tesis de Licenciatura. Por un lado, las temáticas que se aborden dependerán de la línea de investigación que esté desarrollando el profesor guía. Por otro, la postura epistemológica y metodológica, fundamentales en la elaboración de un escrito con fines epistémicos, también obedecerán a opciones que el profesor ha realizado previamente.

Para poder llevar a cabo un proceso de escritura de la tesis se ponen en juego diferentes estrategias de acción/interacción, como se muestra en la figura 5. En primer lugar, los académicos conciben el proceso de escritura de una tesis de Licenciatura en Historia como un trabajo colaborativo, una interacción permanente entre el profesor y el estudiante, lo que permite una revisión y adecuación constantes a las exigencias de dicha actividad (Castelló 2007). Carlino (2003), por su parte, aboga por la colegialidad en la escritura de una tesis, puesto que es uno de los pilares fundamentales para que los escritores no-expertos vayan adquiriendo la experticia que la comunidad requiera. 


\section{Figura 5}

Estrategias de acción/interacción de la codificación axial del fenómeno 'Proceso de escritura de la tesis'

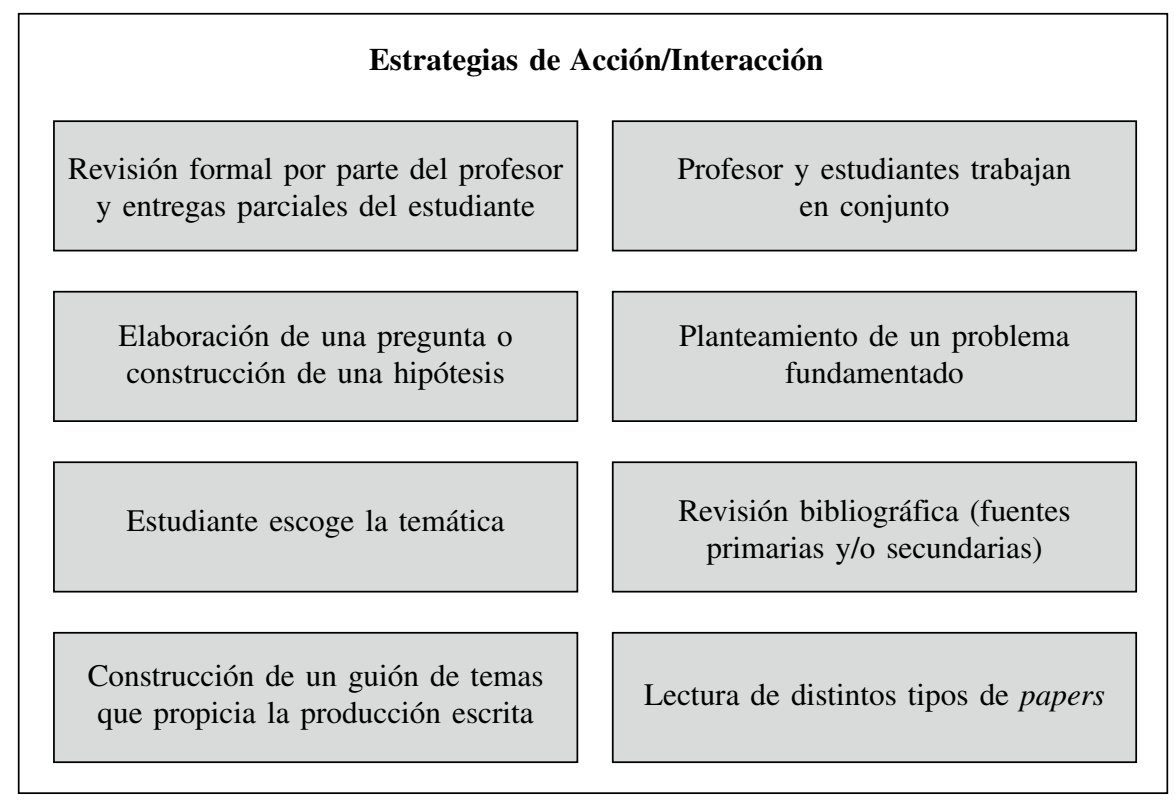

En segundo lugar, respecto de las mismas estrategias de acción/interacción, los entrevistados afirman que los estudiantes deben, al comenzar la escritura de la tesis, plantearse preguntas de investigación o la construcción de hipótesis que guíen la pesquisa historiográfica. Decidirse a plantear una pregunta o una hipótesis dependería del enfoque metodológico del estudio y, también, de las temáticas que se pretendan abordar. Por lo anterior, revisar el tema y plantearse interrogantes, es decir, representarse el problema de escritura pareciera convertirse en la etapa fundamental para comenzar la investigación y, por tanto, la escritura de la tesis.

Al tratarse de una investigación de corte historiográfico o de análisis político, se manifiesta la urgencia por realizar una revisión bibliográfica extensa que nutra al neófito en la materia que pretende discutir. Las fuentes que se revisan pueden ser primarias (textos originales o incunables) o secundarias (textos de divulgación o papers), entre otras.

Para poder dar continuidad al proceso de escritura de la tesis, ambos participantes (profesor guía y tesista) construyen un guión de temas posibles, a modo de índice, que permitirá ordenar la información y propiciará la actividad de escribir. Esta permanente interacción entre investigador y aprendiz no se da solo a nivel conceptual o metodológico, sino también a nivel formal (referencias bibliográficas, gramática y ortografía). En los borradores que los estudiantes entregan a sus profesores, estos últimos señalan que deben oficiar de editores y correctores, ante la poca experiencia del estudiante como escritor de textos académicos. Los procedimientos empleados pueden ser variados ("yo lo hago así, 
me lo envían virtualmente y yo voy haciendo correcciones"), pero el fin es la optimización de la competencia discursiva disciplinar ("cuando van terminando con sus tesis mejoran considerablemente" o "En el ejercicio con uno, [el estudiante] aprende a escribir").

En definitiva, como se aprecia en la figura 6, se aprende a escribir una tesis de Licenciatura en Historia 'por modelo', es decir, mediante la lectura de otros textos con similares propósitos o fines epistémicos (tesis y artículos científicos o papers).

\section{Figura 6}

Consecuencias de la codificación axial del fenómeno

'Proceso de escritura de la tesis'

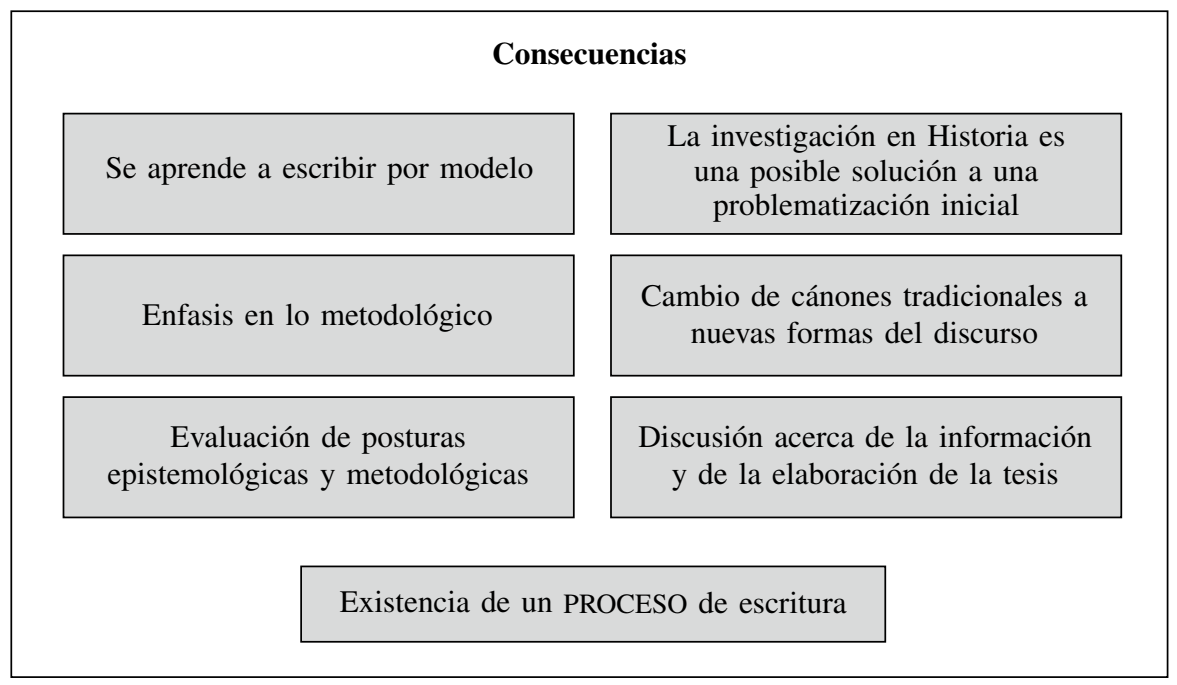

Se caracteriza también este proceso de escritura, en tanto consecuencia del análisis fundado en los datos, por un marcado énfasis en aspectos metodológicos que den consistencia a la investigación. En la búsqueda de dicha consistencia, los cánones tradicionales han derivado en nuevas modalidades discursivas, se pasa de la explicación o la narración a una exposición condensada de información disciplinar. Esto ha supuesto, al interior de la comunidad de especialistas, una discusión acerca de los procesos de elaboración de la tesis. De ahí que se haya delimitado claramente el proceso de escritura del género tesis al finalizar la formación de un licenciado en Historia.

\subsection{HACIA UNA TEORIA FUNDADA EN LOS DATOS}

El fenómeno central de esta investigación fue descrito en dos dimensiones: características y fases. Se puede afirmar, en general, que la escritura de una tesis de Licenciatura en Historia se concibe como un proceso enmarcado en una determinada situación de comunicación de la que tanto el profesor guía como el estudiante tesista son coartífices, aunque en distinto rango. Mientras el profesor es miembro de la comunidad discursiva, el 
estudiante debe realizar el ejercicio de escribir para comenzar a insertarse en ella y, así, subsanar las barreras impuestas por la institucionalidad académica. Tal como se observa en la figura 7, el proceso de escritura de la tesis se concibe como una transacción entre el estudiante y el profesor.

\section{Figura 7}

Esquematización de una teoría emergente respecto del proceso de escritura de una tesis de Licenciatura en Historia

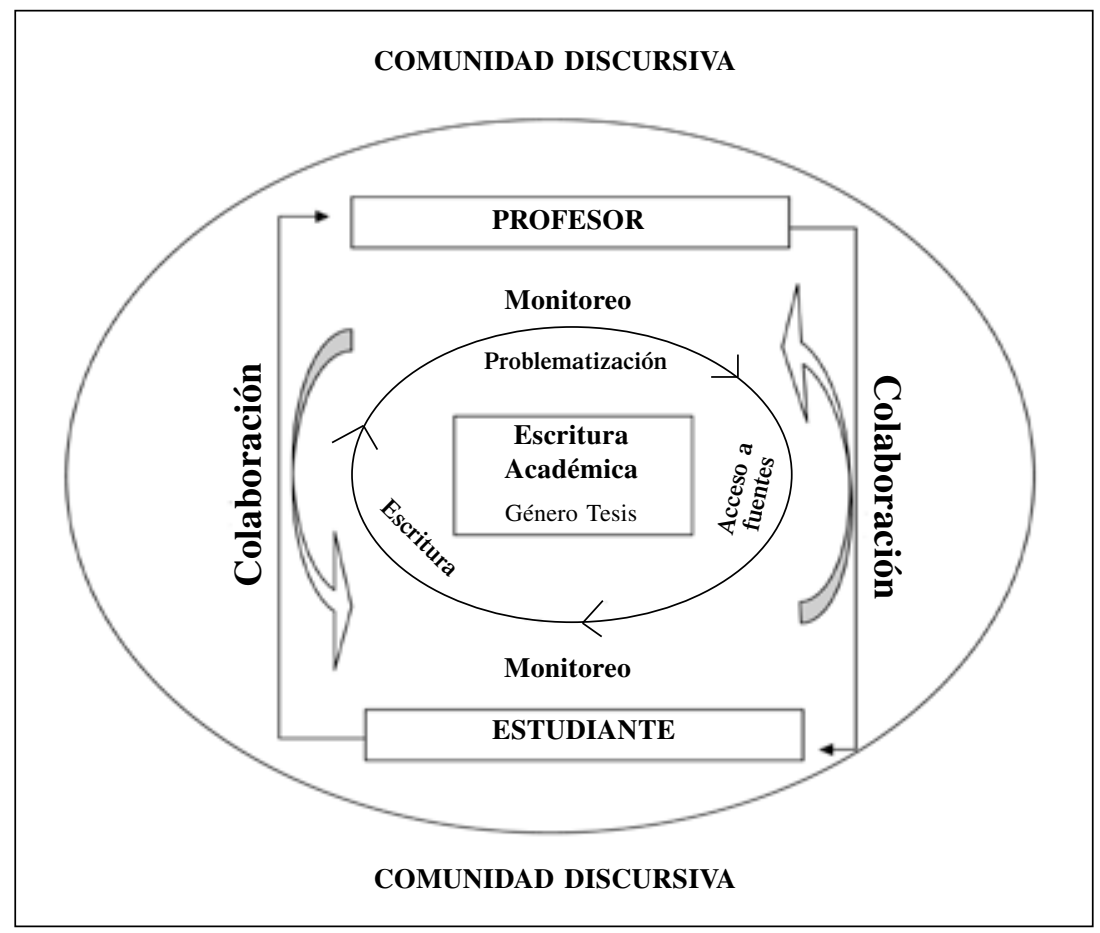

Este proceso de escritura de una tesis, de carácter eminentemente recursivo, se compone de tres fases: Problematización, Acceso a fuentes y Escritura. Respecto de la primera fase, se trata de acceder a una dimensión desconocida, al menos hasta el momento, por el estudiante. Deben ahondar en temáticas particulares y problematizarlas para poder encontrar un nicho desde el cual comenzar la investigación. La segunda fase refleja el conocimiento y las competencias discursivas y disciplinares propias del escritor, su capacidad de decisión, de síntesis de información y de diálogo con la tradición de la especialidad, en que la lectura y la escritura se conforman como actividades propias de la transformación del conocimiento. En esta fase, tal como afirmaran Marinkovich y Morán (1998), en escritura académica, las prácticas del conocimiento y las discursivas son inseparables. Por su parte, la última fase de este proceso corresponde al acto 
composicional en sí mismo del género en cuestión. Se evidencia que la tesis, en tanto género, incorpora las voces de otros textos anteriores y se elabora como respuesta tanto a ellos como a otros que aparecerán posteriormente sobre el mismo tópico (Bajtín, 1982), con un marcado carácter dialógico en su construcción. En esta fase se pasa de la lectura y aprendizaje de conceptos disciplinares a la elaboración de un texto con claros propósitos comunicativos.

Todo el proceso previamente descrito se encuentra bajo el control de un monitoreo permanente en el que el escritor experto revisa y hace consciente de su actividad de producción al escritor semiexperto. En este sentido, no solo se ve mejorado el texto, en su estructura y profundidad metodológica o conceptual, sino que, también, se ve favorecido el estudiante-escritor, ya que va adquiriendo los códigos propios de su comunidad y aprendiendo a decidir qué estrategias son más adecuadas en una determinada fase de la investigación. Tal como afirmara Castelló (2007: 81), "solo sobre la base de los niveles progresivos de representación consciente de las actividades que conlleva la escritura va a ser posible una redescripción de las mismas que permita entender y dominar el complejo proceso de la escritura científica y académica".

En síntesis, las fases de codificación abierta y axial han permitido una aproximación teórica al fenómeno estudiado que respondería a una visión sociocognitiva y discursiva de la escritura académica. Sociocognitiva, porque supone, por una parte, considerar el proceso de escritura como un conjunto de procesos cognitivos por los cuales pasa el escritor para concebir un texto; y, por otra, como un proceso situado socialmente, es decir, dependiente de una situación de comunicación específica. Y, por último, discursiva, porque dicho proceso se realiza según ciertos modos de organización y convenciones que son propios de las diversas comunidades disciplinares y de los géneros discursivos que se producen y circulan en dichos entornos, como es el caso del género tesis en la Licenciatura en Historia (Flower, 1994; Castelló, 2002; Parodi, 2008).

\section{REFLEXIONES FINALES}

No cabe duda de que para describir el proceso de escritura académica y, en ese contexto, la tesis de grado en un nivel inicial de los estudios universitarios, más aún, en una disciplina del área de las Humanidades, como es el caso de la Licenciatura en Historia, no podíamos prescindir de las representaciones sociales que la comunidad de profesores tiene acerca de esta problemática. Más aún, si concordamos en entender las representaciones sociales como el conjunto de puntos de vista y creencias que comparten los miembros de una comunidad sobre algún aspecto de la realidad que los circunda.

Los resultados, producto de un análisis que parte de los datos y vuelve a ellos cuantas veces sea necesario para derivar hipótesis y construir un modelo bajo los principios metodológicos de la TEF, permiten dar cuenta de que en la comunidad explorada el proceso de construir una tesis de grado parte por una problematización tanto de la temática como de las posibilidades de llevar a cabo la investigación, lo que supone desde ya la utilización de la lengua escrita: se revisa y se lee la bibliografía, se toman notas, se realiza un esquema, se elaboran borradores hasta llegar al texto que responda a los propósitos previamente establecidos y en consonancia con la decisión epistemológica y metodológica que exige un trabajo científico. Todo esto en un ir y venir constante no 
solo del alumno consigo mismo, en cuanto al contenido y a la forma, sino también con el profesor que guía el proceso de escribir una tesis de grado.

Esta constatación nos lleva a determinar ciertas regularidades que, a pesar de que solo tienen validez para los casos estudiados, no dejan de ser esclarecedores para la comunidad disciplinar que hemos investigado. En efecto, podemos concluir que, por una parte, la escritura de la tesis en esta especialidad adhiere a dos principios generativos y estructurantes de la escritura como proceso: la recursividad y la dialogicidad. La primera porque implica una resolución de un problema en constante movimiento que toma lo ya escrito para ampliarlo, reelaborarlo y transformarlo en un discurso nuevo, el que, a su vez, generará otros discursos. La segunda porque la práctica de la escritura supone una interacción social entre el Yo, el Tú y el Saber (Martínez, 1997).

Por otra parte, estos principios rectores se complementan con el rigor científico que una tesis en esta área exige, que aunque esté marcada por su naturaleza humanística y social no transa a la hora de aplicar el método que le es propio según los propósitos comunicativos que la orientan.

En cuanto a las limitaciones de este estudio, coincidimos con Strauss y Corbin (1994), en que una investigación de este tipo está siempre sesgada por la interpretación que el investigador otorga a los datos recogidos, más aún, si estos datos son poco heterogéneos, ya que corresponden a una sola disciplina académica.

En suma, estamos ciertos de que lo logrado hasta aquí es un primer intento por acercarnos a la problemática del proceso de escritura de una tesis en un campo específico del saber. Es necesario, entonces, contrastar las representaciones sociales de los profesores con aquellas de los estudiantes acerca del mismo proceso y cómo otras disciplinas del ámbito académico conciben este género propio de la formación inicial para que quienes ya la han finalizado puedan integrarse paulatinamente a una comunidad disciplinar determinada.

\section{REFERENCIAS BIBLIOGRAFICAS}

Albentosa, J. y Moya, A. (2000). "La reducción del grado de transitividad de la oración en el discurso científico en lengua inglesa". Revista Española de Lingüística, 30 (2), 445-468.

Bajtín, M. (1982). Estética de la creación verbal. México: Siglo XXI.

Barthes, R. (2003). El sistema de la moda y otros escritos. Barcelona: Paidós.

Bereiter, C. y Scardamalia, M. (1987). The psychology of written composition. Hillsdale, N.J: Erlbaum.

Bhatia, V. (2002). "A generic view of academic discourse". En Flowerdew, J. (Ed.), Academic discourse (pp. 21-39). Cambridge: Cambridge University Press.

Bourdieu, P. (1997). Las reglas del arte. Barcelona: Anagrama.

Calderón, L. y Bono, M. (2003). Manual para la elaboración de tesis de Licenciatura. Número X, Seminario de Investigación Jurídica, México, D. F.

Camps, A. (2007). "Prólogo: Comunicar en contextos científicos y académicos". En Castelló, M. (Coord.), Escribir y comunicarse en contextos científicos y académicos (pp. 9-12). Barcelona: GRAÓ.

Carlino, P. (2002). "Enseñar a escribir en la universidad: cómo lo hacen en Estados Unidos y por qué”. Revista Iberoamericana de Educación, versión digital, agosto de 2002, OEI [en línea]. Disponible en: ttp: //www.rieoei.org/deloslectores/279carlino.pdf 
Carlino, P. (2003). "Alfabetización académica: Un cambio necesario, algunas alternativas posibles". Educere, Revista Venezolana de Educación, 6 (20), 409-420.

Carlino, P. (2005). Escribir, leer y aprender en la Universidad. Una introducción a la alfabetización académica. Buenos Aires: Fondo de Cultura Económica.

Castelló, M. (2000). "Las concepciones que tienen los estudiantes sobre escritura académica". En Pozo, J. \& Monereo, C. (Coord.), El aprendizaje estratégico (pp. 197-217). Madrid: Santillana.

Castelló, M. (2002). "De la investigación sobre el proceso de composición a la enseñanza de la escritura". Revista Signos, 51-52, 149-162.

Castelló, M. (Coord.) (2007). Escribir y comunicarse en contextos científicos y académicos. Barcelona: GRAÓ.

Castorina, J. (2003). "La ideología de las teorías psicológicas en la educación especial”. En Simón, M., Buscaglia, V. \& Massone, M. (Comps.), Educación de sordos: ¿educación especial y/o educación? Selección de artículos presentados en el congreso MERCOSUR. Buenos Aires: Universidad de Buenos Aires.

Di Stefano, M. (2003). Escritura y producción de conocimiento en las carreras de posgrado. Ponencia presentada en el II Congreso Internacional Cátedra UNESCO Lectura y Escritura. Pontificia Universidad Católica de Valparaíso.

Farr, R. (1984). "Las representaciones sociales". En Moscovici, S. Psicología social II. Pensamiento y vida social. Psicología social y problemas sociales. Barcelona-Buenos Aires-México: Paidós.

Flores-González, L. (2008). "Posiciones y orientaciones epistemológicas del paradigma de la complejidad". Cinta moebio, 33, 195-203.

Flower, L. (1989). "Cognition, context, and theory building". College Composition and Communication, 40, 282-311.

Flower, L. (1994). The construction of negotiated meaning A social cognitive theory of writing. Carbondale, IL: University of Southern Illinois Press.

Giménez, G., Ortiz, F. y Sánchez, M. (2000). "Los sujetos y las prácticas escolares: un acercamiento a las representaciones de los adolescentes respecto de la escritura". Lengua y Literatura: temas de enseñanza e investigación, 1, 161-166.

Glaser, B. (1992). Basics of grounded theory analysis: emergence vs forcing. Mill Valley, Ca.: Sociology Press.

Hermosilla, Y. y Villalobos, A. (2006). "El proceso de formación de un investigador. Vivencias y reflexiones". Investigación y postgrado, 21 (2), 69-101.

Hyland, K. (2002). Teaching and Researching Writing. Harlow, UK: Longman/Pearson Education.

Hyland, K. (2003). Second Language Writing. Cambridge: Cambridge University Press.

Kanoksilapatham, B. (2003). A Corpus-based Investigation of Scientific Research Articles: Linking Move Analysis with Multidimensional Analysis. Tesis doctoral, Georgetown University, Washington, DC.

Jodelet, D. (1984). "La representación social: fenómenos, concepto y teoría". En Moscovici, S. (Ed.). Psicología Social II. Pensamiento y vida social. Psicología social y problemas sociales. Barcelona: Paidós.

Krause, M. (1995). "La investigación cualitativa: Un campo de posibilidades y desafíos". Revista Temas de Educación, 7, 1-22.

Marinkovich, J. y Morán, P. (1998). "La escritura a través del currículum”. Revista Signos, 31 (43-44), 165-171.

Martínez, C. (1997). "El desarrollo de estrategias discursivas a nivel universitario". En Martínez, C. (Comp.), Los procesos de la lectura y la escritura (pp. 11-41). Santiago de Cali: Universidad del Valle. 
Meneses, A. (2008). "Leer y escribir en una escuela chilena: Representaciones discursivas de los diferentes agentes educativos en las áreas prioritarias del currículo escolar en NB3". Signos, 41 (67), 257-278.

Narvaja de Arnoux, E. (2006). "Incidencia de la lectura de pares y expertos en la reescritura de tramos del trabajo de tesis". RLA, 44 (1), 95-118.

Pereira, C. y Di Stefano, M. (2007). "El taller de escritura en posgrado: Representaciones sociales e interacción entre pares". Signos, 40 (65), 405-430.

Paltridge, B. y Starfield, S. (2007). Thesis and dissertation writing in a second language. London: Routledge Falmer.

Parodi, G. (Ed.) (2008). Géneros académicos y géneros profesionales: Accesos discursivos para saber y hacer. Valparaíso: Ediciones Universitarias de Valparaíso.

Petrucci, A. (2002). La ciencia de la escritura. Buenos Aires: Fondo de la Cultura Económica.

Rubio, M. (2008). Evolución de las concepciones sobre el proceso de producción escrita en estudiantes de pedagogía básica durante su formación como docentes. Tesis doctoral, Pontificia Universidad Católica de Valparaíso, Valparaíso, Chile.

Sppigle, H. (1994). "Analysis and interpretation of qualitative data in consumer research". Journal of Consumer Research, 21, 491-503.

Strauss, A. y Corbin, J. (1990). Basics of qualitative research: Grounded theory procedures and techniques. Newbury Park, CA: Sage Publications.

Strauss, A. y Corbin, J. (1994). "Grounded Theory Methodology: an overview". En Denzin, N. y Lincoln, Y. Handbook of Qualitative Research, (pp. 273-285). Londres: Sage.

Strauss, A. y Corbin, J. (eds.) (1997). Grounded Theory in Practice. London: Sage Publications.

Strauss, A. y Corbin, J. (2002). Bases de la investigación cualitativa. Técnicas y procedimientos para desarrollar la teoría fundamentada. Bogotá: Universidad de Antioquia.

Suddaby, R. (2006). "What grounded theory is not". Academy of Management Journal, 49, 633642.

Swales, J. (1981). Aspects of article introductions. Birmingham: University of Aston.

Swales, J. (1990). Genre Analysis: English in Academic and Research Settings. Cambridge: Cambridge University Press.

Swales, J. (2004). Research Genres: Explorations and Applications. New York: Cambridge University Press.

Treet, B. (1999). Academic literacies: position paper. Ponencia presentada en el Simposio New Directions in Literacy Research, Association Internationale de Linguistique Appliquée, Tokyo, Japón.

Vasilachis, I. (1997). Discurso político y prensa escrita: La construcción de las representaciones sociales. España: GEDISA.

Venegas, R. (2006). "La similitud léxico semántica en artículos de investigación científica en español: Una aproximación desde el Análisis Semántico Latente”. Signos, 39 (60), 75-106. 